\title{
Low Energy STEM-EELS Characterization of Primitive Organic Matter and Silicates in the Meteorite LAP 02342
}

\author{
Bradley T. De Gregorio ${ }^{1}$, Rhonda M. Stroud ${ }^{1}$, Larry R. Nittler ${ }^{2}$, Conel M. O’D. Alexander ${ }^{2}$, Jemma \\ Davidson $^{2}$, Carles E. Moyano-Cambero ${ }^{3}$, and Josep M. Trigo-Rodríguez ${ }^{3}$ \\ 1. U.S. Naval Research Laboratory, Materials Science and Technology Division, Washington, DC USA. \\ 2. Carnegie Institution of Washington, Department of Terrestrial Magnetism, Washington, DC USA. \\ 3. Institute of Space Sciences - Institute d'Estudis Espacials de Catalunya, Barcelona, Spain.
}

Information regarding the dust and gas components that were present in the Solar Nebula that eventually became our current Solar System can be gleaned from remnants preserved in the most primitive meteorites and extraterrestrial dust. These samples often contain abundant carbonaceous matter [1], presolar grains [2], and glass with embedded metal and sulfides (GEMS) [3]. Because the grain sizes of these primitive materials are typically $<1 \mu \mathrm{m}$, high resolution analytical techniques such as (scanning) transmission electron microscopy (S/TEM) are necessary to study them. To avoid potential beam damage by the high-energy (100-200 keV) electron beam, studies of organic matter in these samples have used synchrotron-based, "soft" scanning-transmission X-ray microscopy (STXM) and X-ray absorption near-edge structure spectroscopy (XANES), but the spatial resolution $(\geq 25 \mathrm{~nm} / \mathrm{pixel})$ may not be good enough to resolve the finest features. However, the current generation of low energy, aberration-corrected STEM instruments can achieve an energy resolution for electron energy-loss spectroscopy (EELS) approaching that typical of XANES.

We performed a comparative study of STEM-EELS and STXM-XANES on a rare, carbon-rich xenolith trapped within the moderately-altered meteorite LAP 02342 (Figure 1A). An isotopic survey of this xenolith revealed a high presolar grain abundance, at the level of the most primitive interplanetary dust samples [4, 5]. Furthermore, its carbon-rich composition matches the characteristics of primitive micrometeorites believed to come from comets [1]. Two electron-transparent lamellae were extracted from the xenolith using focused ion beam (FIB) methods, one from the xenolith and one crossing the boundary with the surrounding matrix (Figure 1). The lamellae were first analyzed at beamline 5.3.2.2 at the Advanced Light Source, Berkeley, CA. Subsequently, the lamellae were analyzed at greater magnification using the aberration-corrected Nion UltraSTEM at $60 \mathrm{keV}$, tuned to achieve an EELS resolution of $0.32 \mathrm{eV}$ (comparable to the $0.1 \mathrm{eV}$ resolution of the XANES data).

Similar to preliminary STEM studies of the xenolith [4], both FIB lamellae contain abundant organic matter and GEMS grains (Figure 1B, C). The surrounding matrix is predominantly silicate minerals, with pockets of organic matter (Figure 1B). EELS and XANES of this organic matter show spectral features typical of meteoritic organic matter (Figure 2), with pre-edge peaks attributable to aromatic carbon $(285.0 \mathrm{eV})$, ketone functional groups $(286.7 \mathrm{eV})$, and carboxyl functional groups $(288.5 \mathrm{eV})$. However, spectra from matrix organic matter (orange spectra in Figure 2) show a lower abundance of aromatic carbon and a greater abundance of oxygen-bearing functional groups than spectra from xenolith organic matter (blue spectra in Figure 2). This result matches previous work showing that ironbearing silicate materials are less oxidized within the xenolith [6].

Beam damage in this refractory, aromatic-rich organic matter is minimal in the $60 \mathrm{keV}$ EELS data. Spectrum image scans acquired at a dose $<10^{3} \mathrm{e}^{-} / \AA^{2}$ from the same region on different days are nearly 
identical (cf. two orange EELS spectra in Figure 2). Although the EELS data have broader peaks and lower signal-to-noise than the corresponding XANES data, the quality of the EELS data is sufficient to make a valid interpretation of the true functional group chemistry of the sample. Given that STEMEELS can be quicker and more accessible than synchrotron-based STXM-XANES, these data indicate that STEM-EELS may be a more convenient alternative for characterization of the organic functional chemistry of carbonaceous matter in primitive extraterrestrial samples.

\section{References:}

[1] E Dobrică et al, Geochimica et Cosmochimica Acta 76 (2012), pp. 68-82.

[2] E Zinner, Annual Review of Earth and Planetary Sciences 26 (1998), pp. 147-188.

[3] JP Bradley, Science 265 (1994), pp. 925-929.

[4] RM Stroud et al, $79^{\text {th }}$ Annual Meeting of the Meteoritical Society (2016), abstract 6360.

[5] CE Moyano-Cambero et al, Lunar and Planetary Science Conference XLVII (2016), abstract 2537.

[6] BT De Gregorio and RM Stroud, Microscopy and Microanalysis 23 S1 (2017), pp. 2150-2151.

[7] The authors acknowledge funding from the NASA Emerging Worlds Program.
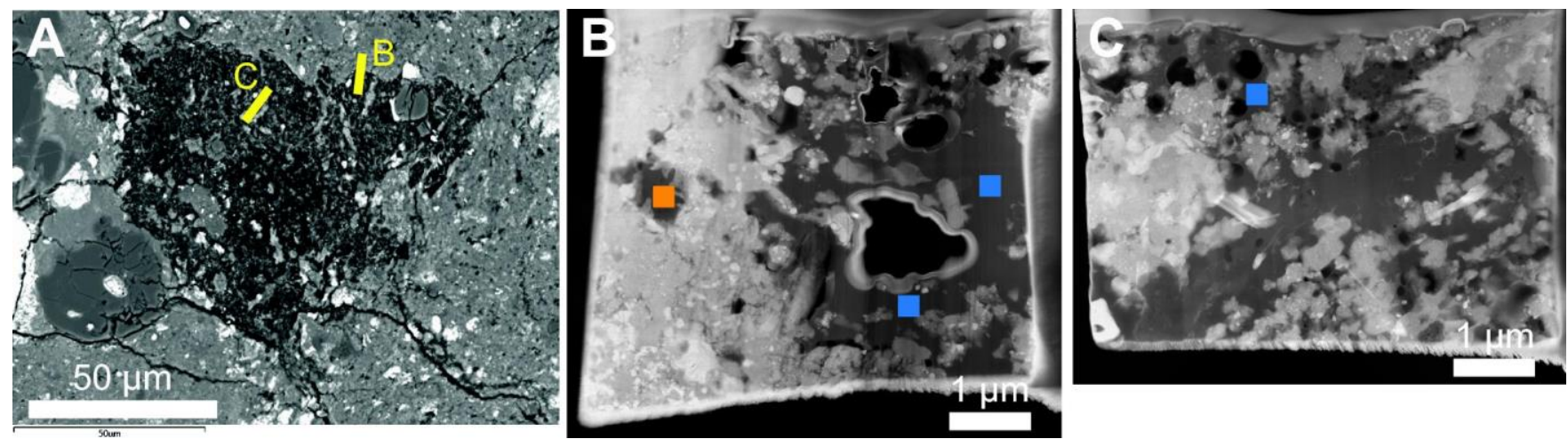

Figure 1. (A) SEM image of the carbon-rich xenolith in meteorite LAP02342. Labeled areas are the locations of the FIB liftout sections. (B) HAADF STEM image of the FIB section crossing the border of the xenolith (right side) and surrounding fine-grained matrix (left side). (C) HAADF STEM image of the FIB section extracted from the xenolith.
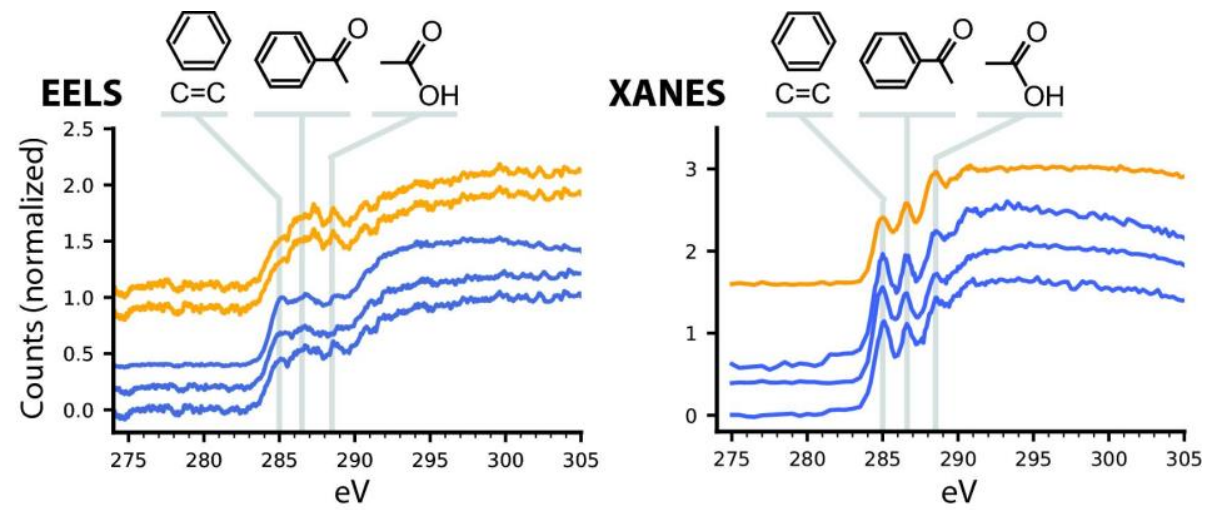

Figure 2. Comparison of carbon EELS and XANES spectra, acquired from the same locations, of organic matter in the xenolith (blue spectra) with that from the surrounding matrix (orange spectra). Analysis locations are denoted on Figures 1B and 1C. EELS spectra have been smoothed and processed to remove instrumental peaks. 